\title{
Design and Implementation of a Safety Statistics Administration System
}

\author{
Jinkui Hou \\ School of Computer Engineering, Weifang University, Weifang 261061, China \\ jkhou@163.com
}

Keywords: E-government system; Information security; Statistics administration

\begin{abstract}
Using of advanced network technology and information technology, a construction scheme for unified statistical information management platform is proposed in this paper. In the scheme, the business of municipal statistics bureau is used as the center, and the scientific statistical system is used as the core. The scheme can be used as the internal service system for the subordinate enterprises and the branch of statistics bureau, which can provide business of online direct report, online surveys, propaganda, consulting, announcements and other statistical information services. The efficiency and accuracy of statistical data reporting are improved, and the labor intensity of statistical work is reduced. This approach can be used to develop a standardized management system covering the whole field of statistical business.
\end{abstract}

\section{Introduction}

As a combination of electronic information technology and management approach, e-government has become the most important areas of the development of information-based modern society. Statistics is an important reference to make decisions for decision-makers. It is also the basis and a powerful tool for understanding and analysis for social economic phenomena. The development of e-government system for statistics department is the need of information construction. It is also the inevitable trend of development of statistical work. The statistical departments must perform statistical functions, which are responsible for statistical data collection, processing, compilation, analysis, and provide services to make decision for government leaders.

In this paper, a construction scheme for unified statistical information management platform is proposed by using of advanced network technology and information technology. The scheme can be used to develop a standardized management system covering the whole field of statistical business.

\section{Analysis of System Requirements}

As an important government agencies, statistics department is responsible for all social statistics, which covering a wide range of data with complex structure. The e-government system of statistics department must follow the basic rules of information technology construction, and the statistical business and the work processes must be restructured and optimized. The platforms for intranet and extranet of e-government are all be built especially.

System functional requirements. The system is used in statistics departments, which should meet the following requirements.

Firstly, the functions of report design and form generation must be strong. The types of the data need to be processed by statistics bureau is complex, and the type of report is quite complex too. Thereby, powerful functions of report design and online forms generation are very important.

Secondly, the form page should provide functions of intuitive data input. The report data of enterprises can be inputted by the way of the same table by hand.

Thirdly, friendly and comprehensive data auditing is capabilities. The system should have a good interface and functions of audit error tracking.

Fourthly, convenient query function should be provided. In the subsystem of direct online reporting, each primary enterprise and institution can query the report and know the progress. Those 
reports failed to pass and returned by the bureau should be processed in time, for, and the examined reports can be queried conveniently.

Fifthly, the system should have powerful functions of summary and data processing. Here, overlay summary and group summary are all supported by the system because the main work of the bureau is summary of reported data.

Sixthly, management of hierarchical rights should be provided by the system. Different departments, enterprises and institutions should be awarded different levels of rights, and unauthorized tampering or leapfrog tampering is prevented.

Seventhly, the system can provide a wealth of information. Beside for the functions of online help to grass-roots enterprises and institutions, there are a variety of experienced reports and comprehensive analysis of the situation, forecast, etc.

Eighthly, multiple formats of export documentation must be supported in the system. Report formats and queried data can be directly exported to the files with the format of Excel, txt, dbf, etc.

Lastly, simple and intuitive interface should be provided by the system. Computer staff at low level can follow the prompts to complete the operation step by step.

Security requirements of the System. The security work should be developed from the aspects of software, hardware and personnel. As for the aspect of software, the main work is the security of server software. Firstly, a rigorous and reasonable access control should be restricted on the file system at the level of operating system, thus the reading and writing of file or program are all strictly controlled. Then, different control rights of application software are marked. One aspect is the control of operator, and the other aspect is the control of operation. For different operators, strict licensing and permissions is granted. Different operators are with different levels of permissions when they login in the system.

In order to ensure the normal operation of the system, the following security requirements are needed to meet.

Authentication. A variety of effective measures must be adopted for authentication through online data transmission, information retrieval and other activities to ensure the legitimacy of user identity. The system must prevent unauthorized users to log maliciously for false reporting.

Access control. The operation rights for users must be restricted to prevent unauthorized access, which include function limits and data access limits. For example, in grass-roots enterprises, the common data reporter, data auditor and the conductor of electronic seals can access different pages. The common data reporter only can input relevant data, and have not the rights to do audit work and stamped work.

Data encryption. Data encryption in the system includes data transmission encryption and storage encryption. XML-based multi-signature encryption algorithm is used in encrypted transmission.

System logs. The information for user accessing is recorded as logs. When abnormal conditions for user logs occur, the information can be queried from the logs.

\section{System design and implementation}

The e-government system of this paper is established for statistical departments, which use B/S architecture, $\mathrm{C} / \mathrm{S}$ architecture and SQL Server database as well as the new generation programming languages (.NET) to design. The B/S architecture is used at the end of clients, all of user functions can be achieved through the browser. The efficiency of deployment and operation of the software system is optimized, and the costs of system operation and maintenance are reduced. Within the management department, the $\mathrm{C} / \mathrm{S}$ architecture is used, which can provide quick and relatively friendly interactive operation. Through technologies of the Internet, direct online reporting is achieved. Advanced security technologies are used to ensure data security and integrity. LAN office and the Internet are combined together to improve work efficiency, and reduce the burden on statistics staff and enterprise.

Overall architecture of the system. The overall system architecture is shown in Fig 1. The users of primary data entry and inner department of statistical bureau can login online direct reporting 
system through the access interface of the platform. The users of grass roots can use the common statistical administrative module, service integration module, and get the electronic seal of the department. The users of inner departments of statistical bureau can use the administrative module, general statistics administration module and service integration module, which can also get the electronic seal of the department at the same time. The users of inner departments of statistical bureau can also access the internal management system to set up users' rights and do data analysis work.

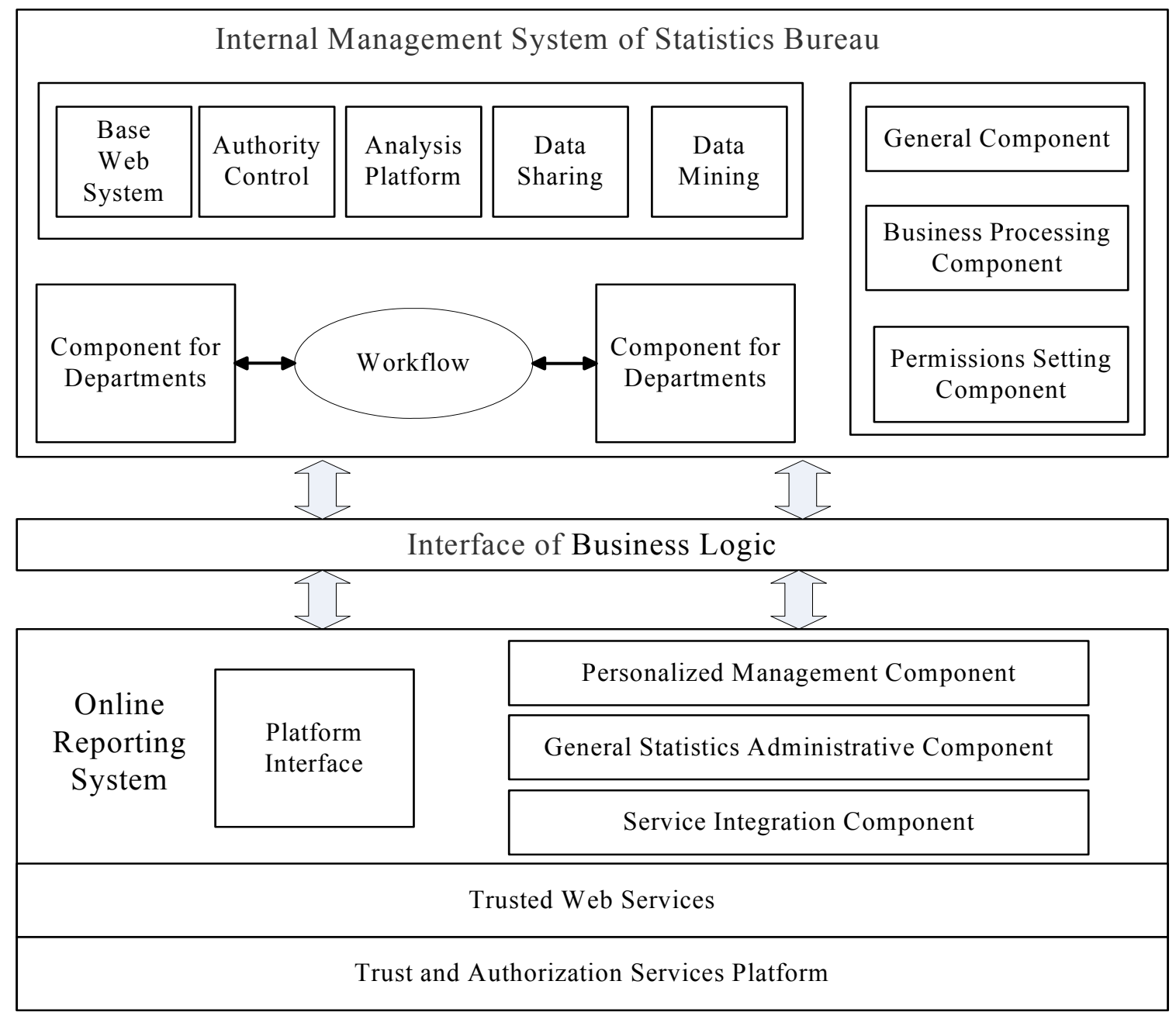

Fig. 1. The overall system architecture

System architecture. The standard 3-tier architecture pattern is used in the internal processing system of the Bureau of Statistics, and the application functions is divided into three-parts, i.e., presentation layer, logic layer and data layer.

The business processing system of the client is the presentation layer, which collects user requests and sends them to the intermediate layer. The final result of data processing should be outputted in the layer. Logic layer is the middle layer, which is responsible for collecting user requests from presentation layer, and then starts business processing. The access request will be sent to data layer, and the business process will end as soon as the data access request is finished. The results will be sent to the presentation layer, and data output is shown on presentation layer. The function of data layer is to perform data request from logical layer, complete data access and send the results to logical layer.

Online reporting system. Online reporting system is based on statistical management center, which establish data acquisition system of primary enterprises and institutions.

The primary data entry department input data through an online page for direct reporting, and the encrypted data will be sent to statistics bureau through the network. The administrator audits the preliminary data and then confirms the data with the stamp of department seal. Finally, the data will be encrypted and be sent to statistics bureau. An XML-based multi-signature algorithm is used in the 
process of data encryption. The user enters the online reporting system. If user login can not pass the authentication, the appropriate description would be given. The users should be determined their identification if passed the authentication, i.e., primary data reporting unit or internal users. The users of grassroots unit can also query statements reported on the unit. The internal users should track grassroots units to give reporting guidance and customs clearance, withdraw unqualified statements and download reported data.

\section{Conclusion}

E-government system is a fundamental research topic with important theoretical significance and broad application prospects. In this paper, an execute solution for safe and efficient services is presented for the e-government system of statistics department, which is on the basis of a large number of related literature and requirement analysis of e-government system. The future work will be concentrated on report design and user interface design to achieve more user-friendly and simplified user interface according to the feedback of users.

\section{Acknowledgments}

The author is most grateful to the anonymous referees for their constructive and helpful comments on the earlier version of the manuscript that helped to improve the presentation of the paper considerably. This research was supported by the foundation of science-technology development project of Shandong Province of China under Grant No. 2011YD01042 and No. 2011YD01043, and the program of science and research reward fund for excellent young scientist of Shandong Province of China under Grant No. BS2011DX039.

\section{References}

[1] B. Hailpern, P. Tarr. Model-driven development: The good, the bad, and the ugly. IBM Systems Journal, Vol. 45, No.3, pp. 451-461 (2010)

[2] J. Miller, J.Mukerji. MDA Guide Version 1.0.1 (Document number omg/20011-06-01). http://www.omg.com/mda (2011)

[3] J. Hou, J. Wan, X. Yang. MDA-based Modeling and Transformation Approach for WEB Applications. In: Proc. of the sixth international conference on Intelligent System Design and Applications (ISDA), pp. 867-812. IEEE Computer Society, New York (2006)

[4] A. Kleppe, J. Warmer, W. Bast. MDA Explained, The Model Driven Architecture: Practice and Promise. Addison-Wesley, Boston (2009)

[5] D. Thomas. MDA: Revenge of the Modelers or UML Utopia? IEEE Software, Vol. 21, No.3, pp. 15-17 (2009)

[6] R. Jeffrey, B. Francesco. Applied Microsoft .NET Framework programming. Microsoft Press, Washington (2009)

[7] T. Mens. A survey of software refactoring. IEEE Trans. on Software Engineering, Vol. 30, No. 2, pp.126-139 (2010) 